\title{
Factores asociados con el tratamiento oportuno de mujeres con cáncer de mama apoyadas por una organización no gubernamental en Bogotá
}

\author{
Guillermo Sánchez¹, Carlos Gustavo Niño', Carolina Estupiñán² \\ 1 División de Investigaciones, Fundación Universitaria de Ciencias de la Salud, Bogotá, D.C., Colombia
}

2 Fundación SIMMON, Bogotá, D.C., Colombia

\begin{abstract}
Introducción. El pronóstico de una mujer con cáncer de mama está relacionado con el tiempo que transcurre desde la aparición de los síntomas hasta el diagnóstico y el tratamiento integral. Las mujeres colombianas enfrentan barreras que afectan su acceso efectivo al sistema de salud.

Objetivo. Establecer los factores determinantes del tratamiento oportuno de un grupo de mujeres con cáncer de mama apoyadas por una organización no gubernamental en Bogotá.

Materiales y métodos. Se llevó a cabo un estudio observacional analítico de 136 mujeres con cáncer de mama apoyadas por una organización no gubernamental de Bogotá. El punto de corte para el tratamiento oportuno se estableció en 90 días calculados a partir de la aparición de los síntomas y hasta el inicio del tratamiento. Se exploraron los factores predictores de oportunidad mediante un análisis multivariado.

Resultados. El $96 \%$ de las mujeres contaba con aseguramiento en salud; el $26,4 \%$ tuvo un tratamiento oportuno, y en 36 casos se negaron los servicios. El $23 \%$ interpuso tutelas para acceder a los servicios de salud. En el análisis multivariado se establecieron asociaciones significativas entre un tratamiento oportuno, el hecho de pertenecer a los estratos socioeconómicos IV y V (razón de probabilidad=3,39), y escolaridad superior a la secundaria (razón de probabilidad=2,72).

Conclusiones. Según la literatura científica, el pronóstico de las mujeres con cáncer de mama se modifica cuando se accede de manera oportuna al tratamiento. En este grupo de mujeres de Bogotá se encontraron factores socioeconómicos que al parecer determinaron su acceso efectivo al tratamiento, lo que revelaría la existencia de 'inequidades' determinadas socialmente.
\end{abstract}

Palabras clave: neoplasias de la mama, accesibilidad a los servicios de salud, investigación sobre servicios de salud, organización social, factores socioeconómicos, Colombia.

doi: http://dx.doi.org/10.7705/biomedica.v35i4.2378

Factors associated with the timely treatment of women with breast cancer supported by a nongovernmental organization in Bogotá, Colombia

Introduction: The prognosis for a woman with breast cancer is related to the time that elapses before diagnosis and integral treatment. Colombian women face barriers that determine effective access to the health system.

Objective: To establish the determinants of timely treatment for breast cancer in a group of women supported by a non-governmental organization in Bogotá.

Materials and methods: An observational analytical study was carried out on 136 women with breast cancer supported by the non-governmental organization. The cut-off point for timely treatment was defined as 90 days, calculated as the time between the appearance of symptoms and the initiation of treatment. Predictors of timely treatment were explored by means of multivariate analysis.

Results: Although $96 \%$ of the women had health insurance only $26.4 \%$ received timely treatment, and 36 of them reported being denied medical services. Of these women, $23 \%$ took legal action to gain access to their healthcare rights. Significant associations were established by multivariate analysis for timely treatment among women belonging to socioeconomic strata IV and V $(\mathrm{OR}=3.39)$, as well as those with higher education (OR=2.72).

\section{Contribución de los autores:}

Guillermo Sánchez: concepción de la idea, seguimiento de la ejecución y análisis

Carlos Gustavo Niño: seguimiento de la ejecución y análisis

Carolina Estupiñán: apoyo en la concepción de la idea, trabajo de campo y recolección de datos

Todos los autores participaron en el diseño del estudio y en la escritura del manuscrito. 
Conclusions: According to the international literature, the prognosis for women with breast cancer improves when they are able to access opportune treatment. In this group of women socioeconomic factors appeared to determine effective access to treatment, revealing the existence of inequalities that may be socially determined.

Key words: Breast neoplasms, health services accessibility, health services research, social organization, socioeconomic factors, Colombia.

doi: http://dx.doi.org/10.7705/biomedica.v35i4.2378

A nivel mundial, el cáncer de mama es la neoplasia más frecuente entre las mujeres, con cifras que ascienden a más de un millón de casos nuevos por año, lo que lo convierte en la primera causa de muerte por cáncer en la población femenina y en un fenómeno creciente en los países en desarrollo.

Según los datos registrados en el proyecto GLOBOCAN de la Organización Mundial de la Salud, la tasa estimada de cáncer de mama para Suramérica está alrededor de 52 casos nuevos por 100.000 mujeres al año; en Colombia, la incidencia anual asciende a 35,7 casos por cada 100.000 mujeres al año (1). Estas cifras contrastan con las tasas de varios países desarrollados, donde la incidencia ha presentado una tendencia al descenso durante los últimos años, probablemente debido a nuevos avances en el diagnóstico y el tratamiento, así como a la implementación de los programas de tamización $(2,3)$.

El pronóstico de la enfermedad está determinado, entre otros factores, por el tiempo entre la aparición de la condición y el acceso oportuno a un tratamiento integral (4-6). En un estudio de población de una cohorte de mujeres noruegas durante nueve años, se concluyó que, al menos, el $33 \%$ del incremento en la supervivencia de las mujeres incluidas en los programas de tamización con mamografía, podía atribuirse a un mejor manejo del cáncer (7). Según los resultados de un estudio en 12 países de 6.588 mujeres, la mayor parte del retraso en el diagnóstico y el tratamiento se debe a demoras propias del sistema de salud, con un promedio de 11,1 semanas (rango: 8,3-24,7), mientras que las demoras atribuidas al paciente solo fueron de 4,7 semanas en promedio (rango: 3,4-6,2) (8). Por ello, la Organización Mundial de la Salud y diversas organizaciones contra el

\section{Correspondencia:}

Carlos Gustavo Niño, División de Investigaciones, Fundación Universitaria de Ciencias de la Salud, Carrera 19 N $8 \mathrm{~A}-32$, Hospital de San José, Bogotá, D.C., Colombia

Teléfono: (571) 353 8100, extensión 3553

cgnino@fucsalud.edu.co

Recibido: 02/05/14; aceptado: 26/05/15 cáncer han planteado que es posible salvar 1,5 millones de vidas que se perderían a causa del cáncer, si se garantizaran el diagnóstico y el inicio del tratamiento oportunos (9). Esta situación se ha confirmado empíricamente, como lo demuestran los datos consignados en la revisión sistemática hecha por Richards, et al., en 1999, en la cual se estableció que la supervivencia era menor cuando el tiempo transcurrido hasta el diagnóstico y el inicio del tratamiento superaba los 90 días (10).

Algunos investigadores han estudiado cómo las demoras en el acceso al diagnóstico y al tratamiento pueden estar socialmente determinadas, con mayor porcentaje de resultados negativos entre la población más vulnerable (11). En el contexto colombiano se han estudiado las causas y los efectos de las demoras en el curso clínico y en el pronóstico del cáncer de mama en las mujeres, y se ha encontrado que hay factores sociales que actúan como barreras de acceso y son responsables de los retrasos en el diagnóstico $y$ en el tratamiento $(12,13)$. Los problemas sociales y las dificultades que enfrenta el sistema de salud colombiano, han propiciado el surgimiento de diferentes organizaciones de apoyo orientadas a facilitar el acceso de los pacientes a los servicios de salud en Colombia.

Con base en el contexto planteado, en la presente investigación se propuso establecer los factores asociados con el tratamiento oportuno del cáncer de mama en un grupo de mujeres apoyadas por una organización no gubernamental en Bogotá.

\section{Materiales y métodos}

Se llevó a cabo un estudio observacional analítico de cohorte retrospectiva basado en la documentación sobre el curso clínico de la enfermedad, entre mujeres con diagnóstico confirmado de cáncer de mama y vinculadas a la Fundación SIMMON de Bogotá entre los años 2011 y 2012.

La convocatoria para el desarrollo del estudio se hizo durante el primer semestre de 2013 a partir de los registros de las bases de datos. Se citaron 190 mujeres para que contestaran el cuestionario de 
recolección de información. De estas, 136 aceptaron participar de forma voluntaria y otorgaron su consentimiento informado. La principal causa para no acudir a la entrevista fue la dificultad en el desplazamiento al sitio de reunión (factores económicos, logísticos y de acompañamiento).

La historia de cada mujer se reconstruyó a partir de fuentes primarias y secundarias, recurriendo a la historia personal, los resúmenes de la historia clínica, los exámenes de laboratorio, las pruebas diagnósticas, las fórmulas médicas y los documentos de presentación de tutelas y otros recursos legales.

El grupo investigador desarrolló un instrumento de recolección de la información que incluía las variables sociodemográficas, los tiempos relacionados con el curso clínico de la enfermedad, la negación de servicios y la interposición de tutelas. El estrato socioeconómico de las mujeres se verificó con el registrado en los recibos de servicios públicos. Se anotaron los ingresos personales y familiares informados en el momento de la encuesta. La variable de negación del servicio de salud se definió como la falta de autorización o de prestación de un servicio requerido por la paciente en el curso de su enfermedad.

Los tiempos de atención se estimaron a partir de los registros verificables. El momento de inicio de los síntomas fue referido por cada participante. Ante la pregunta de la fecha de manifestación inicial, todas las mujeres dieron el mes y el año, y en cuanto al día, se registró el 15 del mes reportado.

Se definió como síntoma inicial cualquier manifestación percibida por la paciente que la llevara a consultar y estuviera asociada con el diagnóstico definitivo de cáncer de mama. Se calcularon los tiempos de atención así: el primer tiempo $\left(T_{1}\right)$ correspondió al transcurrido entre el inicio de los síntomas y la primera consulta médica cuyo motivo principal hubiera estado asociado con el diagnóstico definitivo de cáncer de mama; el segundo tiempo $\left(T_{2}\right)$ se refería al transcurrido entre la primera cita y el diagnóstico (reporte histopatológico confirmatorio de cáncer de mama), y el tercer tiempo $\left(\mathrm{T}_{3}\right)$, el transcurrido entre el diagnóstico y el inicio del tratamiento (conducta terapéutica inicial para el cáncer de mama). El tiempo global $\left(\mathrm{T}_{\mathrm{G}}\right)$ se definió como el transcurrido entre el inicio de los síntomas y el inicio del tratamiento.

Con base en los resultados de Richards, et al., se definió como tiempo oportuno de tratamiento aquel que no superara los 90 días entre las manifestaciones iniciales y el momento de inicio del tratamiento de la enfermedad (10), definición que se empleó para calcular los porcentajes de oportunidad.

En el análisis se emplearon tablas de frecuencia y parámetros estadísticos descriptivos con el fin de ilustrar el comportamiento de las variables. Se exploraron las relaciones existentes entre las características sociodemográficas y la oportunidad en el tratamiento, y se establecieron asociaciones con base en la razón de probabilidades (odds ratio, OR). Con los resultados del análisis bivariado se construyó un modelo multivariado, empleando la técnica de regresión logística. Los análisis se hicieron con el programa estadístico Stata $13^{\circledR}$.

\section{Consideraciones éticas}

El presente proyecto se clasificó como investigación sin riesgo según la Resolución 008430 de 1993 del Ministerio de Salud y fue aprobado por un comité de ética independiente en cumplimiento de las normas nacionales e internacionales para la investigación con seres humanos.

\section{Resultados}

De las 136 mujeres del estudio, 134 residían en Bogotá y dos en los municipios aledaños de Mosquera y Soacha. La edad de las pacientes oscilaba entre los 30 y los 85 años, con un promedio de 57,3 años (desviación estándar, DE=9,9 años), y 129 pertenecían al régimen contributivo 0 al subsidiado (95\%) (cuadro 1).

La mediana del tiempo transcurrido entre el diagnóstico y el inicio del tratamiento fue de 75 días, mientras que la del tiempo transcurrido entre el inicio de los síntomas y el inicio del tratamiento fue de 175 días (figura 1). Con base en los tiempos calculados y según la definición de Richards, et al. (10), el $26,4 \%$ de las mujeres obtuvo un tratamiento oportuno $(36 / 136)$.

Algún servicio de salud le fue negado a 36 mujeres $(26,4 \%)$ durante el proceso de atención (diagnóstico y tratamiento); se negaron en total 58 servicios, es decir, una relación de servicios negados por persona de 1,6. A 20,7 \% (23/111) de las mujeres pertenecientes al régimen contributivo se les negó algún servicio, en tanto que esto le ocurrió al 66,6 \% (12/18) de las mujeres afiliadas al régimen subsidiado. También se le negaron servicios a dos pacientes vinculadas y a una perteneciente a un régimen especial. 
Cuadro 1. Características sociodemográficas de la población de estudio

\begin{tabular}{|c|c|c|c|c|c|c|}
\hline & \multicolumn{2}{|c|}{ Tratamiento oportuno } & \multicolumn{2}{|c|}{$\begin{array}{l}\text { Falta de oportunidad } \\
\text { en el tratamiento }\end{array}$} & \multicolumn{2}{|c|}{ Total } \\
\hline & $n=36$ & $\%$ & $n=100$ & $\%$ & $n=136$ & $\%$ \\
\hline Pareja estable a & 18 & 50,0 & 47 & 47 & 65 & 47,8 \\
\hline Mujeres cabeza de hogar & 10 & 27,8 & 30 & 30 & 40 & 29,4 \\
\hline Vivienda propia & 27 & $75 \ldots$ & 73 & 73 & 100 & 73,5 \\
\hline \multicolumn{7}{|l|}{ Estrato socioeconómico } \\
\hline 1 & 1 & 2,8 & 5 & 5 & 6 & 4,4 \\
\hline 2 & 12 & 33,3 & 42 & 42 & 54 & 39,7 \\
\hline 3 & 12 & 33,3 & 46 & 46 & 58 & 42,7 \\
\hline 4 & 6 & 16,7 & 6 & 6 & 12 & 8,8 \\
\hline 5 & 5 & 13,9 & 1 & 1 & 6 & 4,4 \\
\hline \multicolumn{7}{|l|}{ Escolaridad } \\
\hline Posgrado & 0 & - & 3 & 3 & 3 & 2,2 \\
\hline Universitario & 11 & 30,6 & 8 & 8 & 19 & 14,0 \\
\hline Técnico & 9 & $25 \ldots$ & 13 & 13 & 22 & 16,2 \\
\hline Bachillerato & 8 & 22,2 & 41 & 41 & 49 & 36,0 \\
\hline Primaria & 7 & 19,4 & 31 & 31 & 38 & 27,9 \\
\hline Ninguno & 1 & 2,8 & 4 & 4 & 5 & 3,7 \\
\hline \multicolumn{7}{|l|}{ Tipo de empleo } \\
\hline Formal & 15 & 41,7 & 23 & 23 & 38 & 27,9 \\
\hline Informal & 11 & 30,6 & 28 & 28 & 39 & 28,7 \\
\hline Desempleado & 10 & 27,8 & 49 & 49 & 59 & 43,4 \\
\hline \multicolumn{7}{|l|}{ Ingresos personales } \\
\hline$<1$ SMMLV & 16 & 44,4 & 62 & 62 & 78 & 57,4 \\
\hline 1 a 4 SMMLV & 17 & 47,2 & 37 & 37 & 54 & 39,7 \\
\hline$\geq 4$ SMMLV & 3 & 8,3 & 1 & 1 & 4 & 2,9 \\
\hline \multicolumn{7}{|l|}{ Ingresos familiares } \\
\hline$<1$ SMMLV & 7 & 19,4 & 34 & 34 & 41 & 30,2 \\
\hline 1 a 4 SMMLV & 23 & 63,9 & 59 & 59 & 82 & 60,3 \\
\hline$\geq 4$ SMMLV & 6 & 16,7 & 7 & 7 & 13 & 9,6 \\
\hline \multicolumn{7}{|l|}{ Régimen de afiliación } \\
\hline Contributivo & 33 & 91,7 & 78 & 78 & 111 & 81,6 \\
\hline Subsidiado & 2 & 5,6 & 16 & 16 & 18 & 13,2 \\
\hline Vinculado & 1 & 2,8 & 4 & 4 & 5 & 3,7 \\
\hline Especial & 0 & - & 2 & 2 & 2 & 1,5 \\
\hline Edad promedio & \multicolumn{2}{|c|}{$56,8(\mathrm{DE}=9,2)$} & \multicolumn{2}{|c|}{$57,6(\mathrm{DE}=9,9)$} & \multicolumn{2}{|c|}{$57,9(\mathrm{DE}=9,9)$} \\
\hline
\end{tabular}

a: Mujeres casadas y solteras; SMMLV: salario mínimo mensual legal vigente; DE: desviación estándar

Se establecieron diferencias entre los servicios negados y el tipo de afiliación $(p=0,003)$. En el cuadro 2 se relacionan los principales servicios negados y las causas de la negación.

En el $23 \%$ de los casos se interpusieron tutelas (31/136), con un porcentaje de efectividad del $100 \%$ a los 17 días, en promedio ( $\mathrm{DE}=22$ días).

En el análisis bivariado del diagnóstico oportuno y las variables independientes, se establecieron asociaciones significativas entre pertenecer a un estrato socioeconómico alto (IV $\circ \mathrm{V})$, tener un nivel de escolaridad superior al bachillerato y tener empleo (cuadro 3). No se establecieron diferencias significativas por edad entre los dos grupos $(p=0,82)$. Por último, con base en los resultados del análisis bivariado se estableció un modelo de análisis multivariado de regresión logística y se identificaron como factores predictores significativos el estrato alto (OR ajustada $=3,39, p=0,034$ ), y un nivel de escolaridad superior al bachillerato (OR ajustada $=2,72, p=0,024$ ) (cuadro 4).

\section{Discusión}

En el Sistema General de Seguridad Social en Salud de Colombia (SGSSS), se plantea como una de las principales metas la cobertura universal en el país, y aunque en el año 2013 esta cifra se acercaba al $92 \%$ (14), dicho indicador no reflejaba una situación de acceso real y calidad en la atención. Esto queda demostrado en los resultados del presente estudio: solo el $26,4 \%$ de las mujeres logró acceder a un tratamiento oportuno, con una mediana del tiempo transcurrido desde el inicio de los síntomas hasta el inicio del tratamiento de 175 días (5,8 meses). 


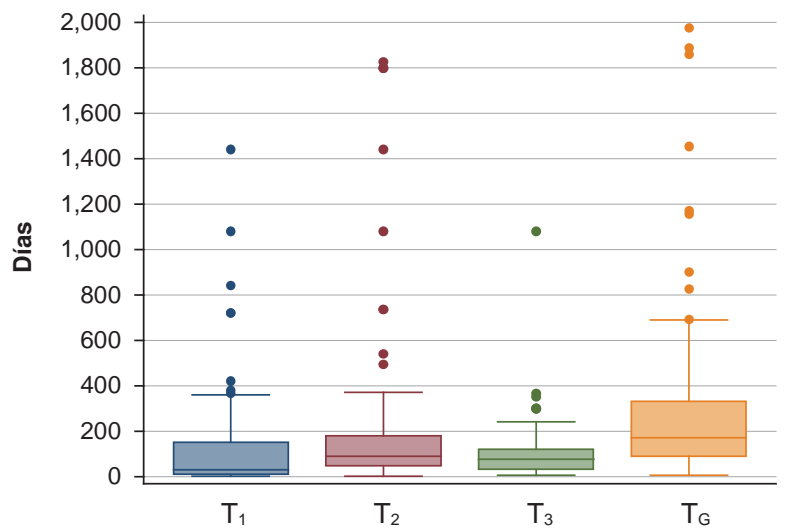

\begin{tabular}{|c|c|c|c|c|}
\hline Tiempos $^{a}$ & $\mathrm{~T}_{1}$ & $\mathrm{~T}_{2}$ & $\mathrm{~T}_{3}$ & $\mathbf{T}_{\mathrm{G}}$ \\
\hline Máximo & 1.440 & $4.015^{b}$ & 1.080 & $4.105^{b}$ \\
\hline Percentil 75 & 120 & 210 & 120 & 330 \\
\hline Mediana & 30 & 90 & 75 & 175 \\
\hline Percentil 25 & 7 & 45 & 30 & 90 \\
\hline Mínimo & 1 & 1 & 5 & 5 \\
\hline \multicolumn{5}{|c|}{$\begin{array}{l}\text { a Tiempos de atención. } \mathrm{T}_{1} \text { : desde el inicio de los síntomas hasta la } \\
\text { primera consulta; } \mathrm{T}_{2} \text { : desde la primera consulta hasta el diagnóstico; } \\
\mathrm{T}_{3} \text { : desde el diagnóstico hasta el inicio del tratamiento; } \mathrm{T}_{\mathrm{G}} \text { : tiempo } \\
\text { global entre el primer síntoma hasta el inicio del tratamiento; }{ }^{b} \text { estos } \\
\text { valores fueron eliminados por su efecto sobre la escala de la gráfica. }\end{array}$} \\
\hline
\end{tabular}

Figura 1. Distribución de tiempos de atención hasta el inicio del tratamiento

Cuadro 2. Servicios negados y razones de dicha negación

\begin{tabular}{lrr}
\hline & $\mathbf{n}$ & $\%$ \\
\hline Servicios negados a & & \\
$\quad$ Citas con especialista & 12 & 20,7 \\
Quimioterapia & 9 & 15,5 \\
Laboratorios & 8 & 13,8 \\
Cirugías & 7 & 12,1 \\
Radioterapia & 7 & 12,1 \\
Imágenes diagnósticas & 6 & 10,3 \\
Medicamentos & 5 & 8,6 \\
Otros procedimientos & 4 & 6,9 \\
Razón de la negación & & \\
Administrativas & 29 & 70,7 \\
No había convenio con la EPS & 8 & 19,5 \\
No se disponía del servicio & 3 & 7,3 \\
El servicio no estaba incluido en el POS & 1 & 2,4 \\
\hline
\end{tabular}

a Se negaron 58 servicios a 36 personas

EPS: empresa prestadora de servicios de salud; POS: plan obligatorio de salud

A pesar de la sentencia T-760 de 2008 proferida por la Corte Constitucional, las barreras de acceso a los servicios de salud en Colombia han obligado a los ciudadanos a buscar en el sistema judicial las herramientas que les permitan alcanzar el goce efectivo del derecho a la salud. Esta situación se hace evidente en el informe presentado por la Defensoría del Pueblo, en el cual se da cuenta de un promedio mensual de 9.596 tutelas por este motivo en 2013 y un total anual de 115.147 (15). Dichos hallazgos están en consonancia con la situación vivida por el $23 \%$ de las mujeres del presente estudio, quienes, a pesar de padecer una enfermedad potencialmente mortal, debieron hacer uso de este recurso legal para acceder de forma efectiva a los servicios de salud que les habían sido negados. Los servicios negados coinciden con lo reportado por la Defensoría del Pueblo en 2014 en torno a las causas que motivaron las tutelas interpuestas en salud en 2013: las peticiones más frecuentes fueron para los tratamientos $(26,18 \%)$, seguidas por la solicitud de medicamentos $(15,92 \%)$, las citas médicas $(11,47 \%)$, las cirugías $(9,41 \%)$, las prótesis, las ortesis y los insumos médicos $(9,06 \%)$, las imágenes diagnósticas $(5,97 \%)$, los exámenes paraclínicos $(4,80 \%)$ y los procedimientos $(1,46 \%)(15)$.

Según su origen, las demoras en el acceso a los servicios de salud se han clasificado así: las relacionadas con el paciente, las de responsabilidad médica y las propias del sistema de salud (16). En el presente estudio se evaluaron estas demoras a partir del cálculo de los tiempos registrados en cada fase de atención, definidos como el periodo transcurrido desde la aparición de los síntomas hasta la consulta inicial $\left(T_{1}\right)$, desde la consulta hasta el diagnóstico $\left(\mathrm{T}_{2}\right)$ y desde el diagnóstico hasta el inicio del tratamiento $\left(\mathrm{T}_{3}\right)$. El tiempo global de atención $\left(T_{G}\right)$ se obtuvo al sumar estos tiempos. Los resultados demostraron que el mayor peso lo obtuvo el periodo $T_{2}$, seguido del $T_{3} y$, en menor proporción, el $\mathrm{T}_{1}$.

En ese sentido, se puede afirmar que en la mayoría de los casos las demoras a las que debieron someterse las mujeres con cáncer de mama son atribuibles a los problemas propios del sistema y que son del resorte de los proveedores de servicios de salud. Estos resultados coinciden con los reportados en el estudio multinacional de Jassem, et al., en el cual la demora en el diagnóstico y el tratamiento se atribuyó en mayor medida al sistema de salud, con un tiempo promedio de 78 días (8). En el contexto latinoamericano, en otros trabajos como el de Trufelli, et al., llevado a cabo en un hospital público brasilero, se encontró que la mediana del tiempo que trascurría entre la sospecha de tumor detectado por mamografía y la biopsia, era de 72 días (17), lo que se aproxima a lo hallado en cuanto al $\mathrm{T}_{2}$ en este estudio. 
Cuadro 3. Análisis bivariado de los factores asociados con un tratamiento oportuno

\begin{tabular}{|c|c|c|c|c|c|}
\hline \multirow[b]{2}{*}{ Factores } & Tratamiento oportuno & $\begin{array}{l}\text { Falta de oportunidad } \\
\text { en el tratamiento }\end{array}$ & \multirow[t]{2}{*}{ OR } & \multirow{2}{*}{$I C_{95 \%}$} & \multirow[t]{2}{*}{$\mathbf{p}$} \\
\hline & n (\%) & n (\%) & & & \\
\hline Pareja estable & $18(50)$ & $47(47)$ & 1,12 & $0,48-2,59$ & 0,75 \\
\hline Vivienda propia & $27(75)$ & $73(73)$ & 1,10 & $0,43-3,03$ & 0,81 \\
\hline Estrato alto a & $11(30,6)$ & $7(7)$ & 5,80 & $1,8-19,4$ & 0,0003 \\
\hline Nivel de estudio alto ${ }^{b}$ & $20(55,6)$ & $24(24)$ & 3,90 & $1,6-9,5$ & 0,0005 \\
\hline Empleado $^{\mathrm{C}}$ & $26(72,2$ & $51(51)$ & 2,49 & $1,02-6,4$ & 0,027 \\
\hline Ingresos personales $>1$ SMMLV & $20(55,6)$ & $38(38)$ & 2,03 & $0,87-4,7$ & 0,067 \\
\hline Ingreso familiar >1 SMMLV & $29(80,6)$ & $66(66)$ & 2,13 & $0,8-6,3$ & 0,1 \\
\hline Régimen contributivo & $33(91,7)$ & $78(78)$ & 3,10 & $0,8-17,1$ & 0,07 \\
\hline
\end{tabular}

a: estratos IV, V y VI; b: formación técnica, universitaria y posgrado; c: incluye empleo formal e informal

SMMLV: salario mínimo mensual legal vigente; OR: razón de probabilidades

Cuadro 4. Regresión logística de los factores predictores de tratamiento oportuno

\begin{tabular}{llcc}
\hline Factores predictores & OR & $\mathbf{p}$ & IC $_{95 \%}$ \\
\hline Estrato alto $^{\mathrm{a}}$ & 3,39 & 0,034 & $1,09-10,47$ \\
Nivel de estudio alto $^{\mathrm{b}}$ & 2,72 & 0,024 & $1,14-6,52$ \\
Régimen contributivo & 2,4 & 0,194 & $0,63-9,05$ \\
\hline
\end{tabular}

a: estratos IV y V

b: formación técnica, universitaria y postgrado

Sujetos correctamente clasificados por el modelo: $77 \%$

OR: odds ratio (razón de probabilidades)

En lo concerniente a los factores que determinan estas demoras, en diversos estudios a nivel internacional se han detectado problemas sociales que pueden generar barreras de acceso y demoras en el proceso de atención $(18,19)$. De hecho, algunos investigadores colombianos han podido establecer que la atención oportuna del cáncer está determinada por la inequidad social $(12,13,20)$. En un estudio de cohorte retrospectiva de 806 mujeres, se concluyó que el acceso al tratamiento no era equitativo y que las mujeres vulnerables enfrentaban mayores demoras (13). En otro estudio, con la participación de 1.240 mujeres con cáncer de mama, se detectaron demoras excesivas e inequidad, especialmente con relación al diagnóstico oportuno (12).

En general, estos estudios plantean que no hay equidad en el acceso y que las mujeres de menores recursos económicos, las de bajo nivel educativo y las afiliadas al régimen subsidiado, son quienes enfrentan las mayores barreras de acceso $(12,13)$. Los resultados del presente estudio apuntan en la misma dirección y señalan que el nivel de escolaridad $(\mathrm{OR}=2,72)$ y el estrato socioeconómico $(\mathrm{OR}=3,39)$ tienen un papel pronóstico importante, lo cual reafirma la tesis de que los factores sociales y su relación con el acceso oportuno a los servicios de salud son determinantes, incluso en los casos de enfermedades catastróficas y potencialmente letales como el cáncer de mama, cuya progresión excede los tiempos administrativos en el marco de un sistema de aseguramiento en salud que no garantiza la equidad en el acceso a los servicios.

Como ya se mencionó, en el estudio de Piñeros, et al., se estableció la asociación con el tipo de afiliación al sistema de salud (12). Sin embargo, en el presente estudio no fue posible establecerla, lo que podría explicarse por el tipo de población de la investigación, dado que, independientemente de su tipo de afiliación, las mujeres que acuden a la ONG lo hacen precisamente porque han tenido que enfrentar barreras de acceso y retrasos en su atención. Por otra parte, vale la pena anotar que en esta investigación solo 18 de las mujeres pertenecían al régimen subsidiado, factor que pudo haber incidido en el hecho de que no se registraron diferencias según el régimen de afiliación.

Diversos investigadores han demostrado cómo las condiciones de salud o enfermedad guardan una estrecha relación con el estatus socioeconómico del individuo, y este, a su vez, está determinado por la estructura política y el modelo de acumulación del capital $(21,22)$. En el caso específico del cáncer de mama, los resultados del presente trabajo permiten apreciar que esta enfermedad no es ajena a la influencia de estos factores. No se está afirmando que el estrato socioeconómico o la educación sean capaces de explicar de manera suficiente la oportunidad en el tratamiento, lo que se plantea es que estos factores hacen parte de una red de elementos que interactúan para garantizar la oportunidad y la calidad en la prestación de los servicios de salud. Algunos autores han estudiado cómo los determinantes socioeconómicos 
de estrato y escolaridad se relacionan con la estabilidad laboral, las tasas de empleo, el poder adquisitivo y la posibilidad de ingresos (23). Está claro que todos estos elementos podrían afectar la oportunidad en el diagnóstico de la enfermedad, sin embargo, estas variables no son más que el producto de una serie de elementos estructurales que determinan este fenómeno. Reconociendo que el acceso a los servicios de salud en las pacientes con cáncer de mama podría estar determinado socialmente, sería pertinente ofrecer un programa integral de atención, sin trámites ni burocracia, en centros especializados que cuenten con los elementos para ofrecer un diagnóstico y un tratamiento oportunos, y que reduzca costos innecesarios para el sistema y mejore el acceso de las pacientes a los servicios de salud.

Es importante señalar que los resultados del presente estudio no pretenden ser un reflejo completo de la situación de las mujeres con cáncer de mama en nuestro país, y que, además, en otros estudios con muestras de mayor tamaño ya se ha abordado este problema (12). De todas maneras, el objetivo era mostrar que, ante las barreras de acceso, algunas mujeres deciden buscar apoyo en organizaciones que contribuyen a disminuir las brechas existentes, así como reafirmar que hay factores sociales que podrían ser responsables de las demoras que retrasan el diagnóstico y el tratamiento. Debe anotarse que existen otros factores potencialmente relacionados con la oportunidad en el diagnóstico y el tratamiento que no fueron abordados en este estudio y podrían ser objeto de futuras investigaciones. Entre ellas vale la pena destacar elementos propios de la psicología de la mujer, así como aspectos específicos de la oferta de servicios y de personal capacitado capaz de enfrentar el reto del cáncer de mama en el país.

La mayor parte de los estudios dedicados al fenómeno de las barreras de acceso tienen que lidiar con potenciales sesgos de recuerdo. El presente estudio no fue la excepción, pero los análisis se basaron en los datos aportados por las mujeres y en la verificación de todas las fuentes posibles, con el fin de aportar el mayor nivel de precisión posible. A pesar de las limitaciones descritas, los resultados obtenidos son importantes para comprender que, a pesar de los avances que el país ha tenido en términos de aseguramiento en salud, estos no son suficientes para garantizar un acceso oportuno y de calidad al sistema de salud, situación que se hace más relevante cuando se enfrenta una enfermedad como el cáncer de mama.
Por último, debe reiterarse que la oportunidad en el acceso y las demoras relacionadas con la atención del cáncer de mama, son producto de la desigualdad social y la inequidad, y que, por lo tanto, la solución implicaría hacer reformas en el sistema de salud como parte de una transformación profunda que contemple garantizar el acceso a la educación y contrarrestar la distribución desigual de la riqueza.

\section{Agradecimientos}

A la Fundación SIMMON, que apoya a pacientes con cáncer, y a su fundadora y presidenta Adriana María Garzón Pinzón, quien en su condición de sobreviviente de cáncer, alentó con su testimonio a otras para participar en este estudio. Agradecemos igualmente el aporte de las mujeres con cáncer de mama que nos contaron su historia.

\section{Conflicto de intereses}

Los autores declaramos que no tenemos conflicto de intereses.

\section{Financiación}

Este proyecto fue financiado con recursos de la convocatoria interna de la Fundación Universitaria de Ciencias de la Salud y de la Fundación SIMMON.

\section{Referencias}

1. International Agency for Research on Cancer. GLOBOCAN 2012: Estimated cancer incidence, mortality and prevalence worldwide in 2012. Fecha de consulta: 6 de mayo de 2015. Disponible en: http://globocan.iarc.fr/ Default.aspx.

2. Berry DA, Cronin KA, Plevritis SK, Fryback DG, Clarke L, Zelen M, et al. Effect of screening and adjuvant therapy on mortality from breast cancer. N Engl J Med. 2005;353:178492. http://dx.doi.org/10.1056/NEJMoa050518

3. Imkampe AK, Bates T. Improvements in breast cancer survival over time, related to adjuvant treatment and node status. Eur J Surg Oncol. 2009;35:151-5. http://dx.doi. org/10.1016/j.ejso.2008.02.010

4. Macleod U, Mitchell ED, Burgess C, Macdonald S, Ramírez AJ. Risk factors for delayed presentation and referral of symptomatic cancer: Evidence for common cancers. Br J Cancer. 2009;101(Suppl.2):S92-101. http:// dx.doi.org/10.1038/sj.bjc.6605398

5. Ramírez AJ, Westcombe AM, Burgess CC, Sutton S, Littlejohns P, Richards MA. Factors predicting delayed presentation of symptomatic breast cancer: A systematic review. Lancet. 1999;353:1127-31. http://dx.doi.org/10.1016/ S0140-6736(99)02142-X

6. Burgess CC, Ramírez AJ, Richards MA, Love SB. Who and what influences delayed presentation in breast cancer? Br J Cancer. 1998;77:1343-8. 
7. Kalager M, Haldorsen T, Bretthauer M, Hoff G, Thoresen SO, Adami HO. Improved breast cancer survival following introduction of an organized mammography screening program among both screened and unscreened women: A population-based cohort study. Breast Cancer Res. 2009;11:R44. http://dx.doi.org/10.1186/bcr2331

8. Jassem J, Ozmen V, Bacanu F, Drobniene M, Eglitis J, Lakshmaiah KC, et al. Delays in diagnosis and treatment of breast cancer: A multinational analysis. Eur J Public Health. 2014;24:761-7. http://dx.doi.org/10.1093/eurpub/ckt131

9. International Agency for Research on Cancer, World Health Organization. Breast cancer screening. Lyon: IARC Press; 2002.

10. Richards MA, Westcombe AM, Love SB, Littlejohns $P$, Ramírez AJ. Influence of delay on survival in patients with breast cancer: A systematic review. Lancet. 1999;353:111926. http://dx.doi.org/10.1016/S0140-6736(99)02143-1

11. Buzdar AU, Smith TL, Powell KC, Blumenschein GR, Gehan EA. Effect of timing of initiation of adjuvant chemotherapy on disease-free survival in breast cancer. Breast Cancer Res Treat. 1982;2:163-9.

12. Piñeros M, Sánchez R, Perry F, García OA, Ocampo R, Cendales R. Demoras en el diagnóstico y tratamiento de mujeres con cáncer de mama en Bogotá, Colombia. Salud Pública Méx. 2011;53:478-85.

13. de Charry LC, Carrasquilla G, Roca S. Equidad en la detección del cáncer de seno en Colombia. Rev Salud Pública. 2008;10:571-82. http://dx.doi.org/10.1590/S012400642008000400007

14. Así vamos en salud. Gráfica - Aseguramiento Georreferenciado. Fecha de consulta: 3 de marzo de 2014. Disponible en: http://www.asivamosensalud.org/ inidicadores/aseguramiento/grafica.ver/15.

15. Defensoría del Pueblo. La tutela y los derechos a la salud y a la seguridad social 2013. Bogotá, D.C.: Imprenta Nacional de Colombia; 2014.
16. Hansen RP, Vedsted P, Sokolowski I, Sondergaard J, Olesen F. Time intervals from first symptom to treatment of cancer: A cohort study of 2,212 newly diagnosed cancer patients. BMC Health Serv Res. 2011;11:284. http://dx.doi. org/10.1186/1472-6963-11-284

17. Trufelli DC, Miranda VD, dos Santos MB, Fraile NM, Pecoroni PG, Gonzaga SD, et al. Analysis of delays in diagnosis and treatment of breast cancer patients at a public hospital. Rev Assoc Med Bras. 2008;54:72-6. http://dx.doi. org/10.1590/S0104-42302008000100024

18. Ibrahim NA, Oludara MA. Socio-demographic factors and reasons associated with delay in breast cancer presentation: A study in Nigerian women. Breast. 2012;21:416-8. http:// dx.doi.org/10.1016/j.breast.2012.02.006

19. Chintamani, Tuteja A, Khandelwal R, Tandon M, Bamal $\mathbf{R}$, Jain S, et al. Patient and provider delays in breast cancer patients attending a tertiary care. JRSM Short Rep. 2011;2:76. http://dx.doi.org/10.1258/shorts.2011.011006

20. Velásquez LC, Carrasquilla G, Roca-Garavito S. Equidad en el acceso al tratamiento para el cáncer de mama en Colombia. Salud Pública Méx. 2009;51(Suppl.2):\$246-53.

21. Moore AD, Hamilton JB, Knafl GJ, Godley PA, Carpenter WR, Bensen JT, et al. The influence of mistrust, racism, religious participation, and access to care on patient satisfaction for African American men: The North CarolinaLouisiana Prostate Cancer Project. J Natl Med Assoc. 2013;105:59-68.

22. Balaam MC, Akerjordet K, Lyberg A, Kaiser B, Schoening $\mathrm{E}$, Fredriksen $\mathbf{A M}$, et al. A qualitative review of migrant women's perceptions of their needs and experiences related to pregnancy and childbirth. J Adv Nurs. 2013;69:1919-30. http://dx.doi.org/10.1111/jan.12139

23. Goddard M, Smith P. Equity of access to health care services: Theory and evidence from the UK. Soc Sci Med. 2001;53:1149-62. http://dx.doi.org/10.1016/S02779536(00)00415-9 